\title{
Combined antitumor gene therapy with herpes simplex virus-thymidine kinase and short hairpin RNA specific for mammalian target of rapamycin
}

\author{
HA-NA WOO ${ }^{1,2^{*}}$, WON IL LEE ${ }^{1,2^{*}}$, JI HYUN KIM $^{1,2}$, JEONGHYUN AHN $^{1,2}$, JEONG HEE HAN $^{3}$, \\ SUE YEON LIM ${ }^{3}$, WON WOO LEE ${ }^{3}$ and HEUIRAN LEE ${ }^{1,2}$ \\ ${ }^{1}$ Department of Microbiology, ${ }^{2}$ Cellular Dysfunction Research Center, University of Ulsan College of Medicine; \\ ${ }^{3}$ Department of Nuclear Medicine, Seoul National University Bundang Hospital, Seoul National University \\ College of Medicine, Seoul, Republic of Korea
}

Received July 31, 2015; Accepted September 25, 2015

DOI: 10.3892/ijo.2015.3194

\begin{abstract}
A proof-of-concept study is presented using dual gene therapy that employed a small hairpin RNA (shRNA) specific for mammalian target of rapamycin (mTOR) and a herpes simplex virus-thymidine kinase (HSV-TK) gene to inhibit the growth of tumors. Recombinant adeno-associated virus (rAAV) vectors containing a mutant TK gene (sc39TK) were transduced into HeLa cells, and the prodrug ganciclovir (GCV) was administered to establish a suicide gene-therapy strategy. Additionally, rAAV vectors expressing an mTORtargeted shRNA were employed to suppress mTOR-dependent tumor growth. GCV selectively induced death in tumor cells expressing TK, and the mTOR-targeted shRNA altered the cell cycle to impair tumor growth. Combining the TK-GCV system with mTOR inhibition suppressed tumor growth to a greater extent than that achieved with either treatment alone. Furthermore, HSV-TK expression and mTOR inhibition did not mutually interfere with each other. In conclusion, gene
\end{abstract}

Correspondence to: Professor Heuiran Lee, Department of Microbiology, University of Ulsan College of Medicine, 88 Olympic-ro 43-gil, Songpa-Gu, Seoul 138-736, Republic of Korea

E-mail: heuiran@amc.seoul.kr

Professor Won Woo Lee, Department of Nuclear Medicine, Seoul National University Bundang Hospital, Seoul National University College of Medicine, 82 Gumi-ro 173 Beon-gil, Bundang-gu, Seongnam-si, Gyeonggi-do, Seoul 463-707, Republic of Korea

E-mail:wwlee@snubh.org

*Contributed equally

Abbreviations: HSV, herpes simplex virus; TK, thymidine kinase; GCV, ganciclovir; shRNA, small hairpin RNA; mTOR, mammalian target of rapamycin; rAAV, recombinant adeno-associated virus

Key words: suicide gene therapy, thymidine kinase, mammalian target of rapamycin, small hairpin RNA, recombinant adenoassociated virus therapy that combines the TK-GCV system and mTOR inhibition shows promise as a novel strategy for cancer therapy.

\section{Introduction}

Gene therapy holds great promise for treating intractable tumors, and suicide gene therapy is one of the most promising strategies (1). The herpes simplex virus (HSV) thymidine kinase (TK) gene is the most widely applied suicide gene in clinical trials (2-5). TK catalyzes the phosphorylation of the prodrug ganciclovir (GCV) to generate the monophosphate form, which is converted by cellular kinases into di- and triphosphate forms that inhibit DNA synthesis (6) and induce apoptosis (7). Furthermore, by exerting the so-called 'bystander effect', toxic metabolites of GCV can kill neighboring non-transfected tumor cells, enhancing cytotoxicity (8). However, the effects of the TK-GCV system are suboptimal, and combination with another strategy is usually recommended $(4,5)$.

Mammalian target of rapamycin (mTOR) is an evolutionally conserved serine/threonine protein kinase and is a target of antitumor therapy because it regulates cell growth and metabolism and also because its aberrant activation occurs in many types of tumors (9-11). In addition to rapamycin, the prototypical inhibitor of mTOR, several rapamycin analogs (rapalogs) show improved pharmacokinetic bioavailability and reduced toxicity (12-14). Moreover, rapalogs such as temsirolimus and everolimus have been approved by the United States Food and Drug Administration (FDA) for treatment of renal cell cancer and lymphoma $(14,15)$. However, the mechanism of mTOR inhibition deserves further scrutiny because many tumors are extremely heterogeneous regarding their sensitivity to rapalogs. This can be explained in part by the complexity of mTOR signaling. mTOR comprises the mTOR complex 1 (mTORC1) and mTOR complex 2 (mTORC2), which interact in a negative feedback loop (11). Thus, inactivation of mTORC1 after the administration of traditional rapalogs leads to paradoxical activation of Akt, the upstream stimulator of mTORC1, by reactivating either the insulin receptor substrate-1 pathway or the mTORC2 pathway, diminishing the antitumor effects 
of a single rapalog (14-16). Further, simultaneous inhibition of mTORC1 and mTORC2 pathways or suppression of all mTORCs and PI3K-Akt pathways using chemical inhibitors is often unacceptably toxic to normal cells despite the improved antitumor effects $(11,14)$. Therefore, a highly sophisticated approach is required if mTOR is to be considered as a therapeutic target for gene therapy.

Here, we developed a novel dual gene therapy strategy and confirmed its potential as a combination gene therapy in a uterine cervical carcinoma model. We employed a suicide gene (HSV-TK) and its prodrug (GCV) to inhibit DNA synthesis and a small hairpin RNA (shRNA) that targeted mTOR expression. Recombinant adeno-associated virus (rAAV) vectors were used for each strategy $(17,18)$. Herein, we show that the combination of the TK-GCV system and mTOR inhibition was efficacious in our model system.

\section{Materials and methods}

Cell culture and reagents. Human cervical carcinoma HeLa cells were obtained from the American Type Culture Collection (Manassas. VA, USA). The cells were maintained in Dulbecco's modified Eagle's medium (Gibco-Invitrogen, Carlsbad, CA, USA) supplemented with $10 \%$ fetal bovine serum (Gibco-Invitrogen), GlutaMAX-1 (2 mM) and $1 \%$ penicillin $(100 \mathrm{IU} / \mathrm{ml}) /$ streptomycin $(50 \mu \mathrm{g} / \mathrm{ml})$ in a humidified atmosphere containing $5 \%$ carbon dioxide at $37^{\circ} \mathrm{C}$.

Construction of a recombinant adeno-associated virus. The rAAV vectors were constructed as previously described $(19,20)$. The rAAV2 plasmid expressing splice-corrected 39TK (sc39TK) under the control of the CMV promoter was constructed by replacing the BamHI-SalI site. The PCR product of the sc39TK gene from pcDNA3.1(+)-sc39TK was inserted into the BamHI-SalI site of the pSp72-self complementary AAV-GFP vector (20). The rAAV2 vectors that expressed mTOR-shRNA or control shRNA driven by an H1 promoter were constructed as described previously (19). The sequences of the mTOR-shRNA were as follows: 5'-GAT CCG AATGTT GAC CAATGC TAT TTC AAG AGA ATA GCA TTG GTC AACATT CTT TTT TGG AAA AGC T-3' (sense) with a BamHI linker and 5'-AGC TTT TCC AAA AAAGAATGT TGA CCA ATG CTA TTC TCT TGA AAT AGCATT GGT CAA CAT TCG-3' (antisense) with a HindIII linker. Nucleotides specific for mTOR are underlined. The generation, preparation, and titration of rAAV2 stocks were performed according to a published method (21).

Cytotoxicity assay. Exponentially growing HeLa cells were seeded in 6-well plates. After overnight incubation, cells were infected with the rAAV2-sc39TK virus at a multiplicity of infection (MOI) of 1,000. At day 1 post-infection, the cells were re-seeded in 96-well plates (10,000 cells per well), and different concentrations of GCV (Cymevene, Roche Diagnostics, Indianapolis, IN, USA) were added to the media, followed by incubation for 4 days. Each treatment condition was tested in triplicate. The CCK-8 assay was performed according to the manufacturer's instructions (Dojindo Molecular Technologies, Inc., Kumamoto, Japan). Cell viability was calculated relative to that of control cells.
Western blot analysis and immunocytochemical analysis. For western blot analysis, proteins were separated using gel electrophoresis through SDS-polyacrylamide gels and then transferred to a PVDF membrane. The membranes were blocked with Tris-buffered saline (TBS) containing $0.1 \%$ Tween-20 and 5\% (w/v) skim milk. After washing with TBST, the membranes were incubated overnight at $4^{\circ} \mathrm{C}$ with antibodies against mTOR (Cell Signaling Technology \#2983, Boston, MA, USA), GFP (Millipore, \#AB16901, Temecula, CA, USA) or TK (from William Summers, Yale University, USA), diluted with TBST containing $1 \%$ skim milk. After washing with TBST, the membranes were incubated for $1 \mathrm{~h}$ at room temperature with the secondary antibodies (Jackson ImmunoResearch Laboratories, Inc., West Grove, PA, USA). Bands were detected using an ECL system (Thermo Scientific, Rockford, IL, USA). For immunocytochemistry, cells were plated in 6-well plates and infected with rAAV2-sc39TK at various MOIs. Cells were blocked with TBS containing $5 \%$ bovine serum albumin (BSA). After washing, the anti-TK antibody (1:200) and Cy3-labeled secondary antibody (1:500) were sequentially added to the fixed cells. Fluorescence signals were analyzed using a fluorescence microscope.

Cell cycle analysis. HeLa cells were infected with either rAAV2-shCont or rAAV2-shmTOR. After $48 \mathrm{~h}$, the cells were trypsinized and fixed with ice-cold ethanol. The cells were then incubated with $0.05 \%$ propidium iodide and analyzed using a flow cytometer (FACSCalibur, Becton-Dickinson, San Jose, CA, USA) and dedicated software (CellQuest, Becton-Dickinson).

Mouse tumor xenograft models. Six-week-old BALB/c nu/nu male mice (Orient Bio Inc., Seongnam, Korea) were used to establish a tumor xenograft model. The institutional animal care and use committee (IACUC) of the Biomedical Research Center at Asan Medical Center approved all procedures involving mice. HeLa cells were infected with rAAV2-shCont, rAAV2-shmTOR, or rAAV2-sc39TK at MOI 1,000. After $24 \mathrm{~h}$ of incubation, $3 \times 10^{6}$ cells were injected subcutaneously into the forelimbs of nude mice. Two weeks later, when the tumors formed by the transplanted cells grew to a volume of $100 \mathrm{~mm}^{3}$, the mice were divided into groups of 4-6, and each mouse was administered one intra-peritoneal injection of GCV $(10 \mathrm{mg} / \mathrm{kg})$ daily for 16 days. Tumor diameter and body weight were measured every 3 days. Tumor volume was calculated as follows: $\mathrm{V}=\left(\mathrm{LxW}^{2}\right) \times 0.5$, where $\mathrm{V}=$ volume, $\mathrm{L}=$ length, and $\mathrm{W}=$ width. The mice were sacrificed at designated times, and the tumors were harvested and subjected to further analysis.

Data presentation and statistical analysis. Data were reported as the mean \pm standard deviation (SD). Differences between experimental groups and controls were determined using the Mann-Whitney test and were considered significant at two-tailed $\mathrm{p}<0.05$.

\section{Results}

Recombinant adeno-associated virus vectors. The expression of the GFP or mutant TK (sc39TK) genes was controlled by the constitutive promoter (pCMV) in the rAAV2-GFP or 
A

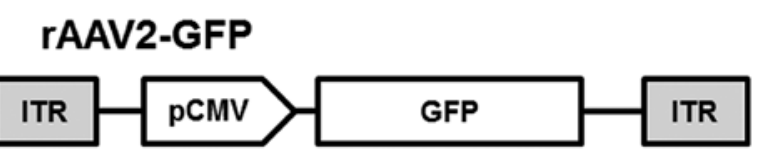

rAAV2-sc39TK

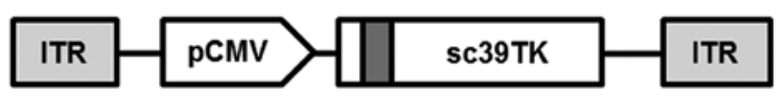

\section{B}
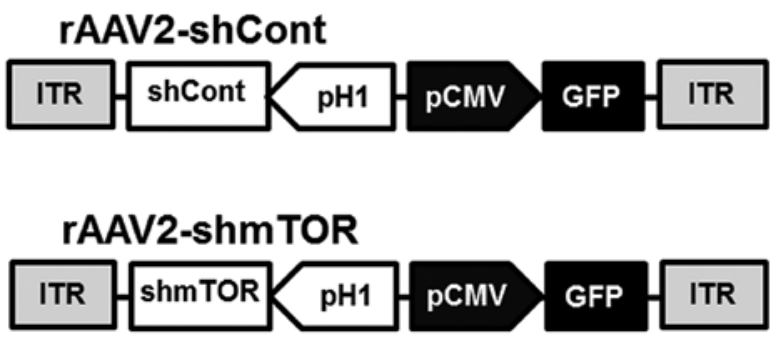

Figure 1. Characteristics of rAAV vectors. (A) rAAV2-GFP and rAAV2sc39TK. The expression of genes encoding the GFP and mutant TK (sc39TK) was under control of the CMV promoter. (B) rAAV2-shCont and rAAV2shmTOR. The control shRNA and the mTOR-specific shRNA were inserted into the rAAV2 cassettes, and their expression was driven by the $\mathrm{H} 1$ promoter. The GFP gene under control of the CMV promoter was used as a reporter gene for expression of the corresponding shRNAs.

rAAV2-sc39TK vectors, respectively (Fig. 1A). The sc39TK gene was generated by introducing a five-codon substitution and a silent mutation in the GCV-resistant splice acceptor and donor sequences (22). These mutations enhance the drug sensitivity of tumor cells $(20,22)$. In contrast, the expression of the control shRNA (shCont) and the mTOR-shRNA (shmTOR) was regulated by the $\mathrm{H} 1$ promoter in the rAAV2-shCont and the rAAV2-shmTOR vectors, respectively (Fig. 1B). The GFP gene controlled by the CMV promoter was inserted as a reporter to detect the expression of the corresponding shRNAs. The mTOR-shRNA was designed using our custom siRNA screening algorithm (Convenient Application Program for siRNA Design, CAPSID), which identifies highly specific siRNAs with minimal off-target effects $(19,23)$.

Suicide gene therapy using the sc39TK-GCV system. We first determined the transduction efficiency of rAAV2-sc39TK for HeLa cells at various MOIs. The control vector (rAAV2GFP) transduced $\sim 90 \%$ of the HeLa cells at a MOI 1,000, and this rate was recapitulated in the cells infected with rAAV2-sc39TK. More than $90 \%$ of the cells expressed sc39TK after infection with rAAV2-sc39TK at MOI 1,000, which was used in subsequent experiments (Fig. 2A). Western blot analysis demonstrated that induction of sc39TK expression (Fig. 2B) continued up to 8 days after infection, which was the last time-point for the in vitro experiments. To determine the cytotoxicity of the sc39TK-GCV system, HeLa cells infected with rAAV2-sc39TK were treated with various concentrations of GCV. There was a concentration-dependent reduction in the cell viability of the rAAV2-sc39TK-infected cells (Fig. 2C). The relative viability of the rAAV2-sc39TK-infected cells decreased to $0.52 \pm 0.15$ at $0.05 \mu \mathrm{g} / \mathrm{ml} \mathrm{GCV}$ and was completely abolished at $1 \mu \mathrm{g} / \mathrm{ml} \mathrm{GCV}$ (Fig. 2C). In xenografted mice, cells infected with rAAV2-sc39TK without GCV treatment continued to grow as tumors until day 29 after engraftment, whereas tumor formation was impaired when the cells were treated with GCV. This effect was apparent at day 26, and the differences in tumor volume on day 29 were statistically significant $(\mathrm{p}<0.05)$ (Fig. 2D). Therefore, the results indicate efficient transduction of HeLa cells with rAAV2-sc39TK and effective tumor inhibition after GCV administration.

mTOR inhibition using shRNA. The transduction efficiency of the rAAV2-shRNA vectors was assessed by determining the levels of GFP expression at various MOIs. At MOI 1,000, $\sim 90 \%$ of HeLa cells expressed GFP, and the expression levels of rAAV2-shmTOR-infected cells were equivalent to those of rAAV2-shCont-infected cells (Fig. 3A). The expression of mTOR was specifically inhibited in cells infected with rAAV2-shmTOR (Fig. 3B). The suppression of mTOR expression in vitro continued up to 8 days after infection with the rAAV2-shmTOR vectors. The in vitro cellular response to mTOR inhibition was determined by analyzing the cell cycle. Infection with rAAV2-shmTOR notably increased the proportion of cells in the G1 phase and decreased the proportion of cells in the $\mathrm{S}$ and $\mathrm{G} 2 / \mathrm{M}$ phases. The proportion of rAAV2shmTOR-infected cells in the G1 phase increased by $8.4 \%$ relative to the proportion in cells infected with rAAV2-shCont (Fig. 3C). In xenografted mice, the volume of tumors induced by engrafted cells infected with rAAV2-shmTOR was significantly reduced compared with those induced by cells infected with rAAV2-shCont (Fig. 3D).

Combination gene therapy using the sc39TK-GCV system and mTOR inhibition. To investigate the effects of combined gene therapy, the mice were subjected to the treatments as follows: i) rAAV2-shCont, ii) rAAV2-shCont with GCV, iii) rAAV2-sc39TK and rAAV2-shmTOR without GCV, and iv) rAAV2-sc39TK and rAAV2-shmTOR with GCV. On day 17, tumors generated by cells infected with rAAV2-shmTOR and rAAV2-sc39TK were smaller than those generated by cells that were infected with rAAV2-shCont (Fig. 4A). Administration of GCV further reduced the growth of tumors generated by cells co-infected with rAAV2-sc39TK and rAAV-shmTOR, and the tumor volumes were smallest in this group (Fig. 4A). On day 29 after implantation, compared with the group of mice engrafted with cells infected with rAAV2-shCont, there was a decrease in tumor volume of $27.9 \pm 6.3 \%$ in the group not treated with GCV but implanted with cells co-infected with rAAV2-shmTOR/rAAV2-sc39TK. Furthermore, there was a $56.8 \pm 8.2 \%$ decrease in tumor volume in the group treated with GCV and implanted with cells co-infected with rAAV2shmTOR/rAAV2-sc39TK (Fig. 4B). On day 29, western blot analysis revealed maintained TK expression and suppressed mTOR expression in tumors formed by cells co-infected with rAAV2-shmTOR/rAAV2-sc39TK (Fig. 4C). The gross size of the dissected tumors formed by cells co-infected with rAAV2shmTOR/rAAV2-sc39TK was smaller than that of tumors formed from cells infected with rAAV2-shCont. Addition of GCV further reduced the size of the tumors of the former group (Fig. 4D). 
A

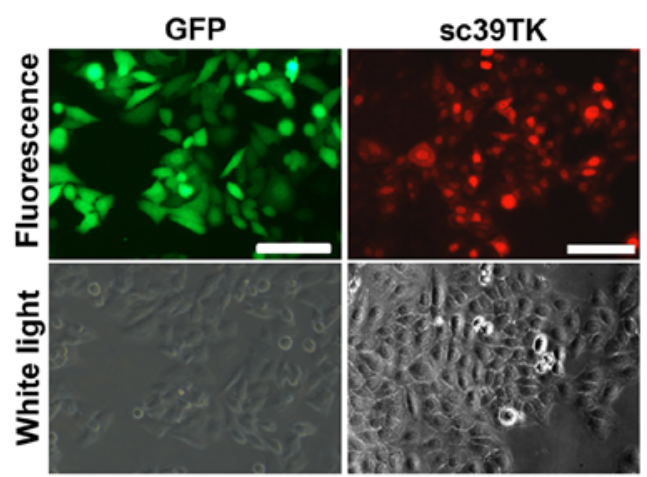

C

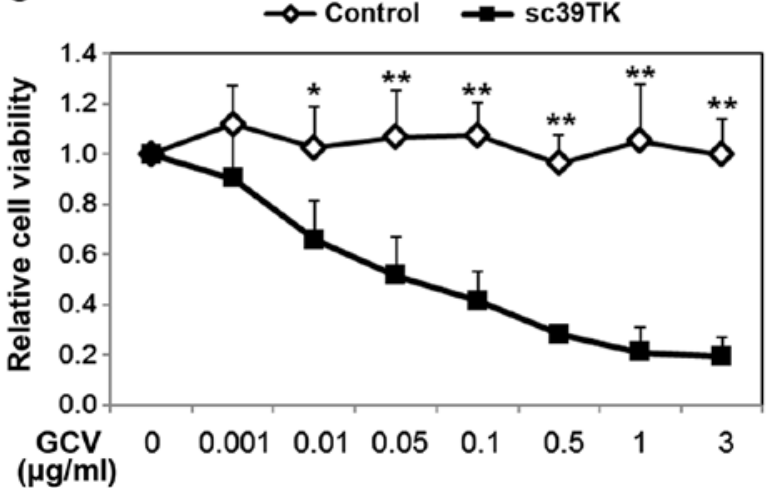

B

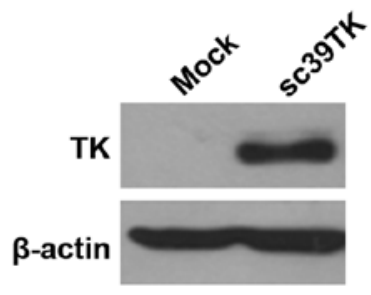

D

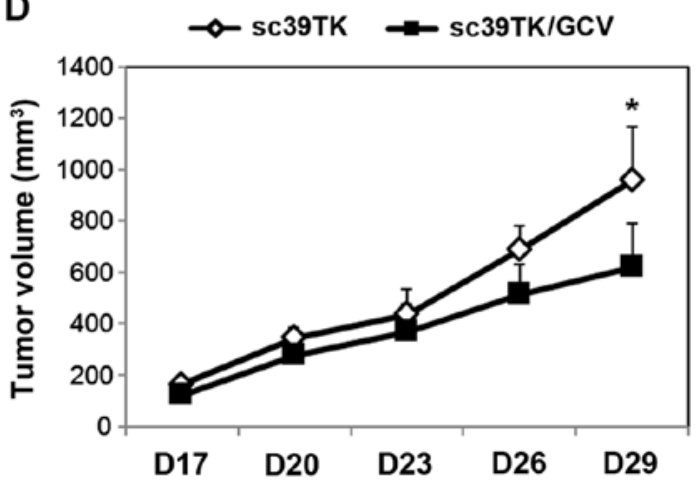

Figure 2. Suicide gene therapy using the sc39TK-GCV system. (A) Transduction efficiency of rAAV2-GFP or rAAV2-sc39TK in HeLa cells at MOI 1,000. Scale bars, $100 \mu \mathrm{m}$. (B) Western blot analysis of TK expression $48 \mathrm{~h}$ after infection of HeLa cells with rAAV2-sc39TK. $\beta$-actin served as a loading control. (C) Cytocidal effects on HeLa cells by sc39TK expression $96 \mathrm{~h}$ after GCV treatment. (D) Effects of GCV on tumors expressing sc39TK in xenografted mice. GCV was injected intraperitoneally every day from day 14 to $29 .{ }^{*} \mathrm{p}<0.05 .{ }^{* *} \mathrm{p}<0.005$. $\mathrm{n} \geq 3$.

A

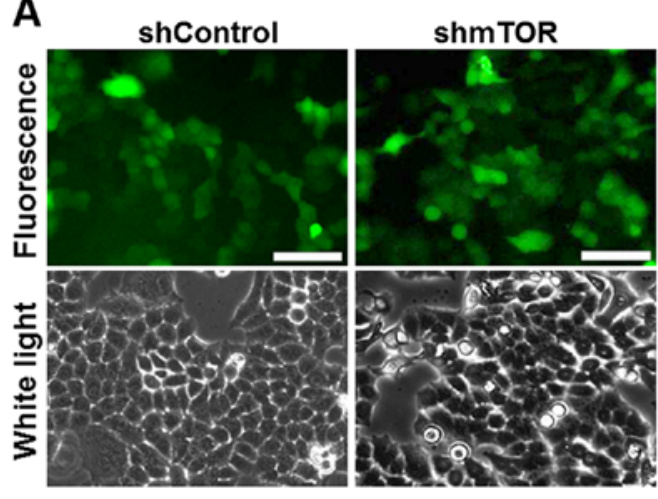

C

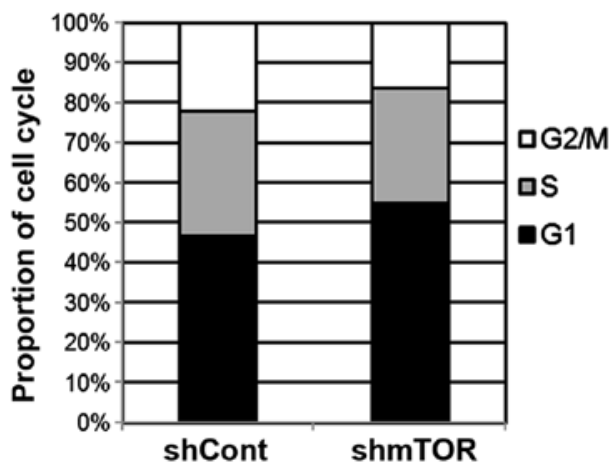

B

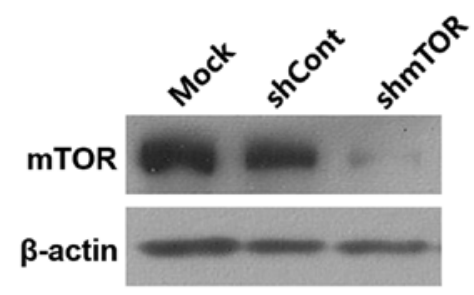

D

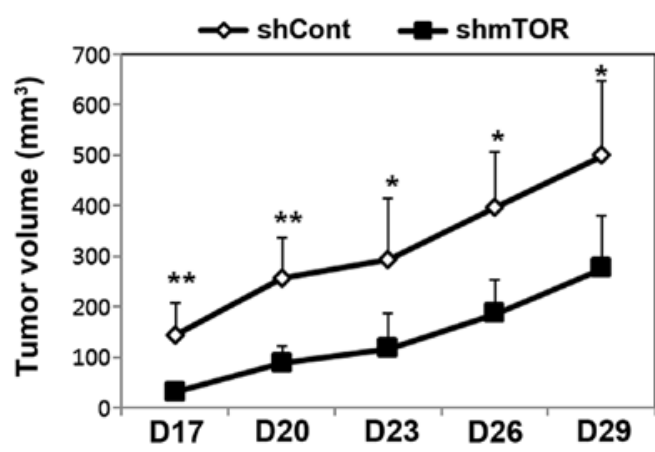

Figure 3. mTOR inhibition using rAAV-mediated RNAi. (A) Equivalent transduction efficiency 48 h post-infection (MOI 1,000) in HeLa cells transduced with rAAV2-shCont or rAAV2-shmTOR. Scale bars, $100 \mu \mathrm{m}$. (B) Western blot analysis for mTOR inhibition by rAAV2-shmTOR. $\beta$-actin served as a loading control. (C) Cell cycle analysis of HeLa cells infected with rAAV2-shmTOR or rAAV2-shCont at $48 \mathrm{~h}$ post infection. (D) Analysis of tumor growth in mice xenografted with HeLa cells expressing shmTOR. ${ }^{*} \mathrm{p}<0.05 .{ }^{* *} \mathrm{p}<0.005 . \mathrm{n} \geq 3$. 
A

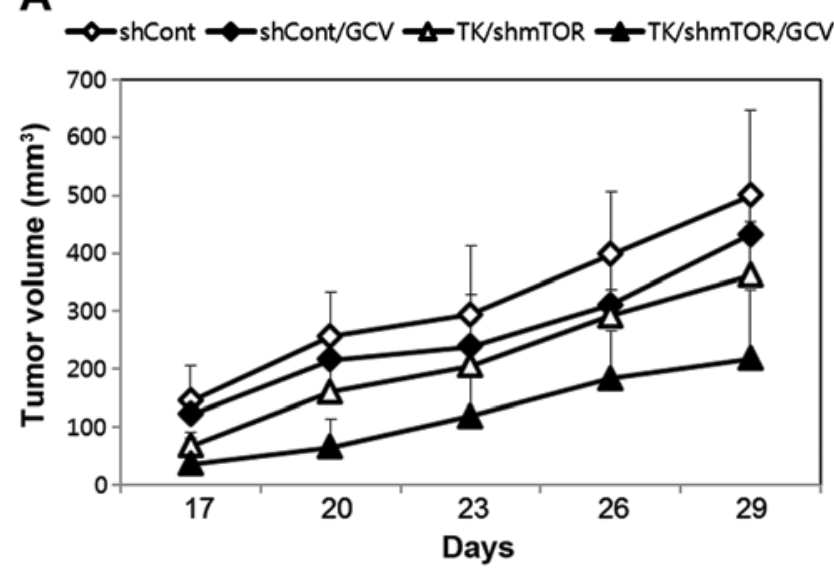

C

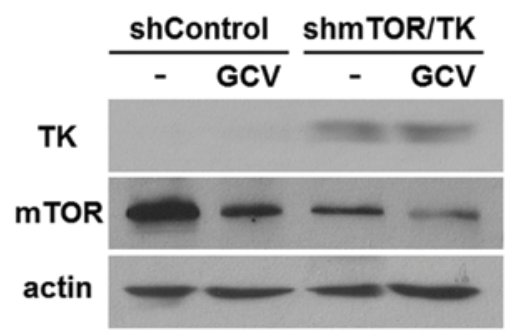

B

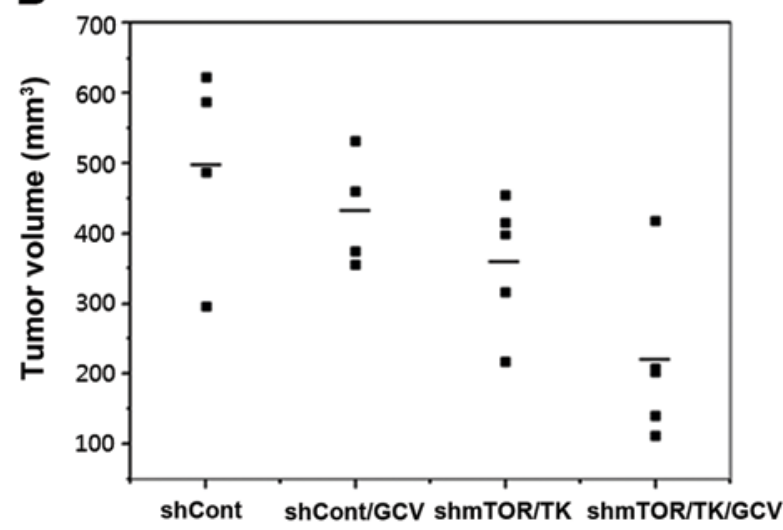

D

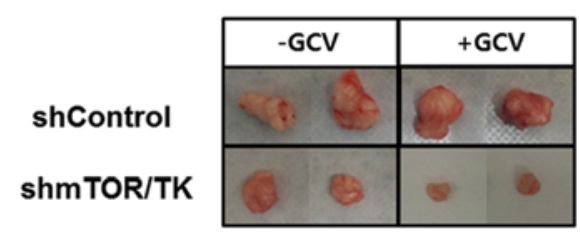

Figure 4. In vivo validation of combination therapy with the sc39TK-GCV system and mTOR inhibition in xenografted mice. HeLa cells were infected with the rAAVs at MOI 1,000. (A) Volumes of tumors according to the treatments. (B) Tumor volumes of each experimental group on day 29. The horizontal bars indicate the mean tumor volumes of each group. (C) Western blot analysis of TK and mTOR expression at the end of the experiment on day 29. (D) Representative images of tumors showing the effects of the sc39TK-GCV system and mTOR inhibition.

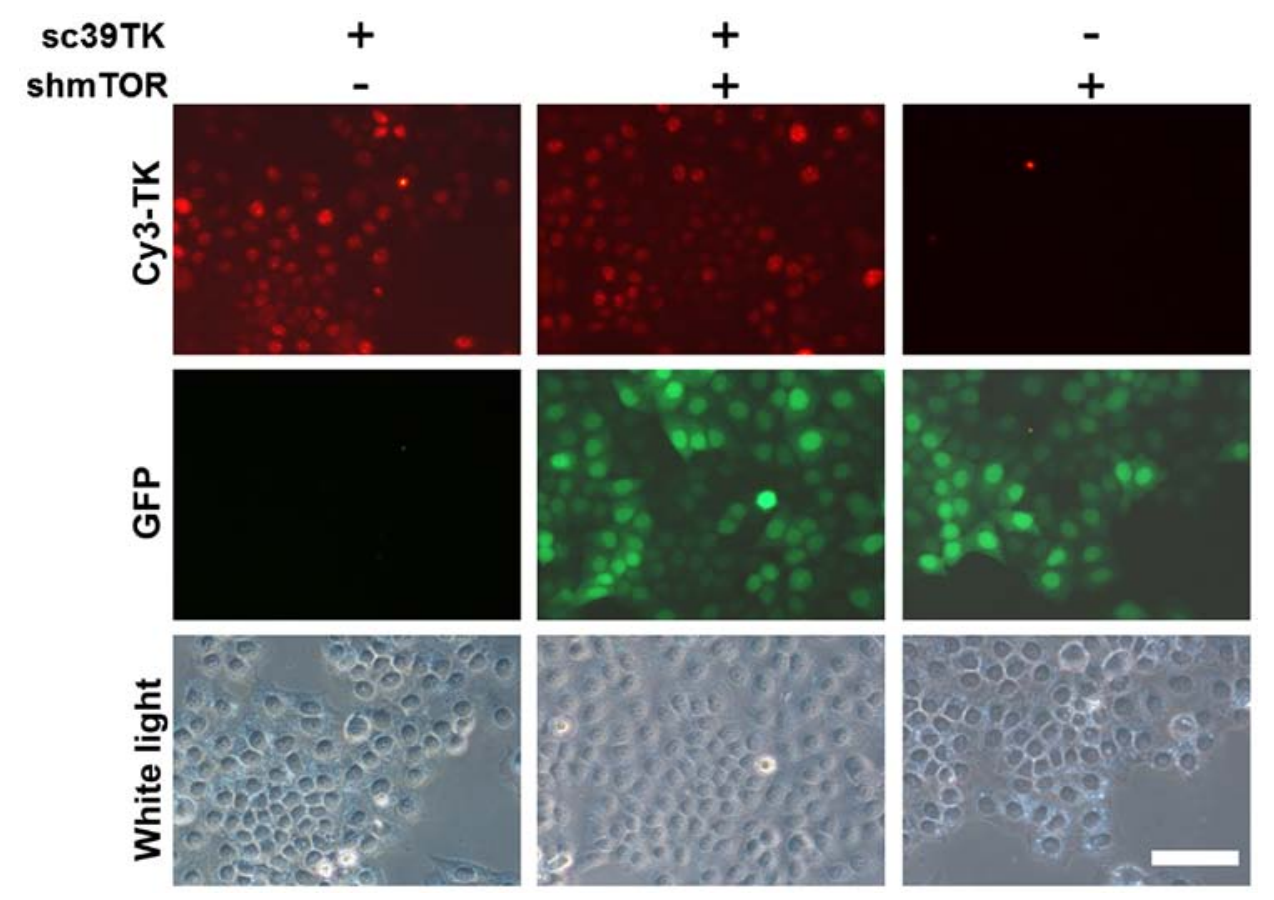

Figure 5. Lack of mutual interference of transgene expression in the co-infected cells. Cells were infected with rAAV2-sc39TK and/or rAAV2-shmTOR at MOI 1,000. GFP signals represent the expression of shmTOR. sc39TK expression was determined by immunocytochemistry as described in Fig. 2A. Scale bar, $100 \mu \mathrm{m} . \mathrm{n} \geq 2$.

Effects of combination gene therapy on transgene expression. We further investigated whether combined transduction of cells with rAAV2-sc39TK and rAAV2-shmTOR mutually inhibited the expression of the transgenes. It is of note that there is no GFP signal from rAAV2-sc39TK (Fig. 1A). In the HeLa cells infected with both rAAV2-sc39TK and rAAV2-shmTOR, the 
GFP signals were comparable to those from rAAV2-shmTOR alone (Fig. 5). Furthermore, TK expression by cells infected with rAAV2-sc39TK was not inhibited by co-infection with rAAV2-shmTOR. Moreover, when the cells were infected at MOI $\leq 5,000$, neither the GFP signal nor TK expression level was reduced (data not shown).

\section{Discussion}

In the present study, we demonstrate for the first time to our knowledge that the combination of suicide gene therapy (sc39TK-GCV system) and mTOR inhibition using an mTORspecific shRNA enhanced antitumor effects without mutual interference of transgene expression. Taking into consideration that the tumors increase in size because of proliferation (increase of cell number) and growth (increase of cell size) of the individual tumor cells, the cytocidal effects of the sc39TK-GCV system (Fig. 2) combined with the cytostatic effect of mTOR inhibition (Fig. 3) serve as an ideal antitumor mechanism (24). The sc39TK-GCV system effectively inhibits the proliferation of tumor cells by inhibiting DNA synthesis $(1,6,20)$, whereas the mTOR-specific shRNA inhibits the growth of tumor cells by downregulating tumorigenic pathways (19).

The TK-GCV system has been evolved in many aspects. The original version of HSV-TK was modified to sc39TK to enhance its antitumor effects upon GCV treatment $(20,22,25)$. A theranostic (simultaneous therapy and diagnosis) approach for individualized therapy was successfully achieved using TK activity that incorporates therapeutics such as GCV and diagnostic imaging agents such as ${ }^{18} \mathrm{~F}-\mathrm{FHBG}$ (a radiolabeled penciclovir analog for positron emission tomography) $(4,20)$. However, because the therapeutic efficacy of the TK-GCV system alone is usually insufficient $(4,5)$, the TK-GCV system has been tested in combination with other antitumor strategies such as another suicide gene, conventional chemotherapy, radiotherapy, and immunotherapy (1). In fact, the combination here of TK-GCV with RNAi is a relatively new approach to cancer therapy (26).

mTOR is an emerging target of antitumor gene therapy because it functions in the PI3K/Akt/mTOR signaling pathway that mediates tumorigenesis $(13,27)$. mTOR regulates the growth, senescence, survival, and metabolic homeostasis of cells (11). Elevated mTOR activity is present in many tumors, and suppression of mTOR induces tumor regression $(9,10)$. Moreover, gene therapy targeting mTOR is a promising antitumor approach, because, as an evolutionally conserved target, relatively few mutant forms are known $(13,28)$. However, mTOR inhibitors have been found to be successful for treating only a few types of tumors such as renal cell carcinoma or lymphoma, and the responses of many other tumors to such drugs are highly heterogeneous (13). In this regard, the RNAi approach used in the present and previous studies shows promise because mTOR-shRNA inhibits both mTORC1 and mTORC2 without serious off-target effects $(19,23)$.

Using rAAV as a vector for gene therapy may have contributed substantially to the success of the combination approach. Wide applicability to a variety of tumors and long-term expression of transgenes without serious toxicity are advantages of using rAAV vectors $(29,30)$. Furthermore, rAAV has been FDA-approved for gene therapy $(29,31)$. We have used rAAV vectors for transgene packaging, delivery, and expression and for in vitro and in vivo monitoring in our previous studies (20,32-34). In the present study, the transduction efficiencies were well balanced between the HSV-TK gene and the mTOR-shRNA (Figs. 2 and 3). Transgene expression was well maintained until the end of the experiments (Fig. 4) without mutual interference (Fig. 5). The demonstration of the combined effects of the dual gene therapy strategy might have been difficult without this expertise of rAAV vectors.

However, some questions remain unanswered regarding the utility of the dual gene therapy presented here. In the TK-GCV system, actively dividing tumor cells are killed directly, and the non-dividing quiescent tumor cells may be indirectly affected by toxic GCV metabolites through the bystander effect. Whether or not the bystander effect contributes to tumor inhibition in conjunction with mTOR inhibition is unknown (8). Additionally, the present study does not address techniques for delivery to patients or the effects of treatment for $>1$ month (3). These questions require further study.

In the present proof-of-concept study, we demonstrate that the TK-GCV system combined with an mTOR-specific shRNA enhances the suppression of tumor growth compared to the use of either technique alone. This promising result may lead to the development of a novel gene therapy strategy against intractable tumors.

\section{Acknowledgements}

This study was supported by grants from Medical Research Center Program (2008-0062286 to H. Lee), Basic Science Research Program (NRF-2011-0014821 to H.N. Woo), and Nuclear Research and Development Program (NRF-2014M2B2A9030104 and NRF-2012M2A2A7035589 to W.W. Lee), Republic of Korea.

\section{References}

1. Duarte S, Carle G, Faneca H, de Lima MC and Pierrefite-Carle V: Suicide gene therapy in cancer: Where do we stand now? Cancer Lett 324: 160-170, 2012

2. Immonen A, Vapalahti M, Tyynelä K, Hurskainen H, Sandmair A, Vanninen R, Langford G, Murray N and Ylä-Herttuala S: AdvHSV-tk gene therapy with intravenous ganciclovir improves survival in human malignant glioma: A randomised, controlled study. Mol Ther 10: 967-972, 2004.

3. Rainov NG: A phase III clinical evaluation of herpes simplex virus type 1 thymidine kinase and ganciclovir gene therapy as an adjuvant to surgical resection and radiation in adults with previously untreated glioblastoma multiforme. Hum Gene Ther 11: 2389-2401, 2000.

4. Sangro B, Mazzolini G, Ruiz M, Ruiz J, Quiroga J, Herrero I, Qian C, Benito A, Larrache J, Olagüe C, et al: A phase I clinical trial of thymidine kinase-based gene therapy in advanced hepatocellular carcinoma. Cancer Gene Ther 17: 837-843, 2010.

5. Voges J, Reszka R, Gossmann A, Dittmar C, Richter R, Garlip G, Kracht L, Coenen HH, Sturm V, Wienhard K, et al: Imagingguided convection-enhanced delivery and gene therapy of glioblastoma. Ann Neurol 54: 479-487, 2003.

6. Moolten FL: Tumor chemosensitivity conferred by inserted herpes thymidine kinase genes: Paradigm for a prospective cancer control strategy. Cancer Res 46: 5276-5281, 1986.

7. Wei SJ, Chao Y, Hung YM, Lin WC, Yang DM, Shih YL, Ch'ang LY, Whang-Peng J and Yang WK: S- and G2-phase cell cycle arrests and apoptosis induced by ganciclovir in murine melanoma cells transduced with herpes simplex virus thymidine kinase. Exp Cell Res 241: 66-75, 1998. 
8. Mesnil $\mathrm{M}$ and Yamasaki H: Bystander effect in herpes simplex virus-thymidine kinase/ganciclovir cancer gene therapy: Role of gap-junctional intercellular communication. Cancer Res 60: 3989-3999, 2000.

9. Bjornsti MA and Houghton PJ: The TOR pathway: A target for cancer therapy. Nat Rev Cancer 4: 335-348, 2004.

10. Liu Q, Thoreen C, Wang J, Sabatini D and Gray NS: mTOR Mediated Anti-Cancer Drug Discovery. Drug Discov Today Ther Strateg 6: 47-55, 2009

11. Zoncu R, Efeyan A and Sabatini DM: mTOR: From growth signal integration to cancer, diabetes and ageing. Nat Rev Mol Cell Biol 12: 21-35, 2011.

12. Cornu M, Albert V and Hall MN: mTOR in aging, metabolism, and cancer. Curr Opin Genet Dev 23: 53-62, 2013.

13. Faivre S, Kroemer G and Raymond E: Current development of mTOR inhibitors as anticancer agents. Nat Rev Drug Discov 5 : 671-688, 2006

14. Fasolo A and Sessa C: Current and future directions in mammalian target of rapamycin inhibitors development. Expert Opin Investig Drugs 20: 381-394, 2011.

15. Pópulo H, Lopes JM and Soares P: The mTOR signalling pathway in human cancer. Int J Mol Sci 13: 1886-1918, 2012

16. Sun SY, Rosenberg LM, Wang X, Zhou Z, Yue P, Fu H and Khuri FR: Activation of Akt and eIF4E survival pathways by rapamycin-mediated mammalian target of rapamycin inhibition. Cancer Res 65: 7052-7058, 2005

17. Hermonat PL and Muzyczka N: Use of adeno-associated virus as a mammalian DNA cloning vector: Transduction of neomycin resistance into mammalian tissue culture cells. Proc Natl Acad Sci USA 81: 6466-6470, 1984.

18. Park K, Kim WJ, Cho YH, Lee YI, Lee H, Jeong S, Cho ES, Chang SI, Moon SK, Kang BS, et al: Cancer gene therapy using adeno-associated virus vectors. Front Biosci 13: 2653-2659, 2008

19. Ahn J, Woo HN, Ko A, Khim M, Kim C, Park NH, Song HY, Kim SW and Lee H: Multispecies-compatible antitumor effects of a cross-species small-interfering RNA against mammalian target of rapamycin. Cell Mol Life Sci 69: 3147-3158, 2012.

20. Kim JY, Kim JH, Khim M, Lee HS, Jung JH, Moon DH, Jeong S and Lee H: Persistent anti-tumor effects via recombinant adeno-associated virus encoding herpes thymidine kinase gene monitored by PET-imaging. Oncol Rep 25: 1263-1269, 2011.

21. Shin O, Kim SJ, Lee WI, Kim JY and Lee H: Effective transduction by self-complementary adeno-associated viruses of human dendritic cells with no alteration of their natural characteristics. J Gene Med 10: 762-769, 2008
22. Black ME, Kokoris MS and Sabo P: Herpes simplex virus-1 thymidine kinase mutants created by semi-random sequence mutagenesis improve prodrug-mediated tumor cell killing. Cancer Res 61: 3022-3026, 2001

23. Lee HS, Ahn J, Jun EJ, Yang S, Joo CH, Kim YK and Lee H A novel program to design siRNAs simultaneously effective to highly variable virus genomes. Biochem Biophys Res Commun 384: 431-435, 2009.

24. Schmelzle T and Hall MN: TOR, a central controller of cell growth. Cell 103: 253-262, 2000

25. Black ME, Newcomb TG, Wilson HM and Loeb LA: Creation of drug-specific herpes simplex virus type 1 thymidine kinase mutants for gene therapy. Proc Natl Acad Sci USA 93: 3525-3529, 1996.

26. Park SY, Lee W, Lee J and Kim IS: Combination gene therapy using multidrug resistance (MDR1) gene shRNA and herpes simplex virus-thymidine kinase. Cancer Lett 261: 205-214, 2008.

27. Bartholomeusz C and Gonzalez-Angulo AM: Targeting the PI3K signaling pathway in cancer therapy. Expert Opin Ther Targets 16: 121-130, 2012.

28. Grabiner BC, Nardi V, Birsoy K, Possemato R, Shen K, Sinha S, Jordan A, Beck AH and Sabatini DM: A diverse array of cancerassociated MTOR mutations are hyperactivating and can predict rapamycin sensitivity. Cancer Discov 4: 554-563, 2014.

29. Mingozzi $F$ and High KA: Therapeutic in vivo gene transfer for genetic disease using AAV: Progress and challenges. Nat Rey Genet 12: 341-355, 2011.

30. Mueller $\mathrm{C}$ and Flotte TR: Clinical gene therapy using recombinant adeno-associated virus vectors. Gene Ther 15: 858-863, 2008.

31. Ylä-Herttuala S: Endgame: Glybera finally recommended for approval as the first gene therapy drug in the European union. Mol Ther 20: 1831-1832, 2012.

32. Kim SJ, Lee WI, Heo H, Shin O, Kwon YK and Lee H: Stable gene expression by self-complementary adeno-associated viruses in human MSCs. Biochem Biophys Res Commun 360: 573-579, 2007.

33. Kim SJ, Lee WI, Lee YS, Kim DH, Chang JW, Kim SW and Lee H: Effective relief of neuropathic pain by adeno-associated virus-mediated expression of a small hairpin RNA against GTP cyclohydrolase 1. Mol Pain 5: 67, 2009.

34. Lee HS, Shin OK, Kim SJ, Lee WI, Jeong S, Park K, Choe H and Lee $\mathrm{H}$ : Efficient gene expression by self-complementary adenoassociated virus serotype 2 and 5 in various human cancer cells. Oncol Rep 18: 611-616, 2007. 